\title{
DETERMINAZIONE DEL VALORE MEDIO DELLO SPES- SORE DELLO STRATO DI GRANITO PER I TRAGITTI TURKESTAN-EUROPA
}

Pravecsco Peronaci

I. noto dhe uno dei metodi per la deterninazione dello spessore delle stratificazioni della crosta terrestre e basato sulla dispersione ielle onde superficiali rewistrate at distanzal suffeientenente grande affinche leftetlo della dispersione divenga sensibile. Naturalmente il metodo conduce al valore medio dello spersore dello strato in esame mentre per la determinazione eflettiva in una regione sufficientemente ristretla. : necessario ricorrere all'impiego di metoli basati sui tempi di tragitto delle onde fraziali longitudinali e trasserwali. Il primo viene quindi usato quanto non si abbiano dati di registrazione per le regioni prosime all'epicentro.

Utilizzanto le regiatrazioni degli osservatori europei in occasione del forte terremoto del Turkestan del 2 novembre 1946 ho escenito un tentalion di determinazione dello spessore dello strato del granito per i vari tragitti Turkestan-linopa Centrale serventoni delle onde di Love. la cui regitrazione per alcume stazioni is statal particolarmente ampia. La teoria di queste onde nella lorma siluppiata da Jelleress consente inlatti la determinaxione dello spessore degli strati -uperficiali of ua a d 10 siano noti la velociti di propalazione $c_{n}$ e $c^{\prime}$

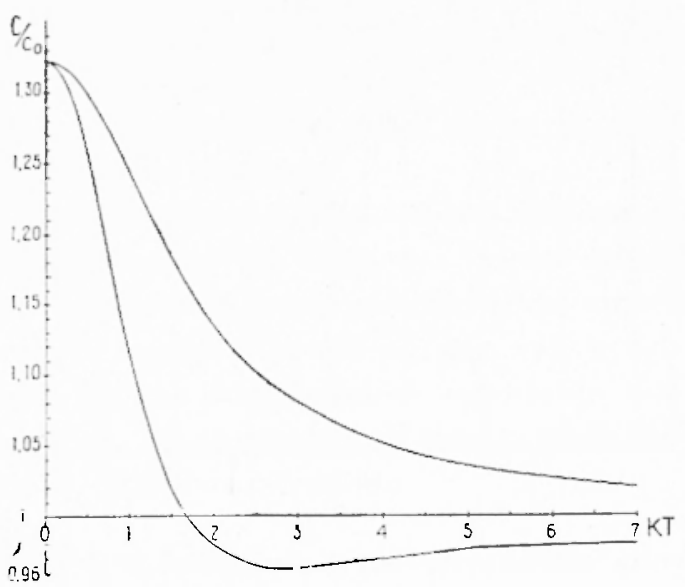

Fig. 1 telle onde tratsersali spaziali alla superficie superiore ed inferiore dello strato e la densiti $e_{0}$ " $g^{\prime}$ della prima e seconda stratificazione nellipotesi particolare di stratificazioni onogenee. 
Tal teoria infalli permelte di traceiare, se a e C sono le velociti di lase e di gruppo delle onde considerate e d lo spessore dello slralo, 16 curve che lorniscono l'andamento dei rapporti $c / c_{1}$ e $C$ c in lunzione di $\frac{2 \pi d}{\lambda}$ quamdo si tenga presente la relazione intereedente fra le relocila di gruppo e di lise

$$
c=c-\hat{\lambda} \frac{\partial c}{\partial \lambda}
$$

Non ritengo neessatio riasumere sia pur breremente il meloslo analitico da seguire perehe noto. limitamdomi a fare osservate rhe una

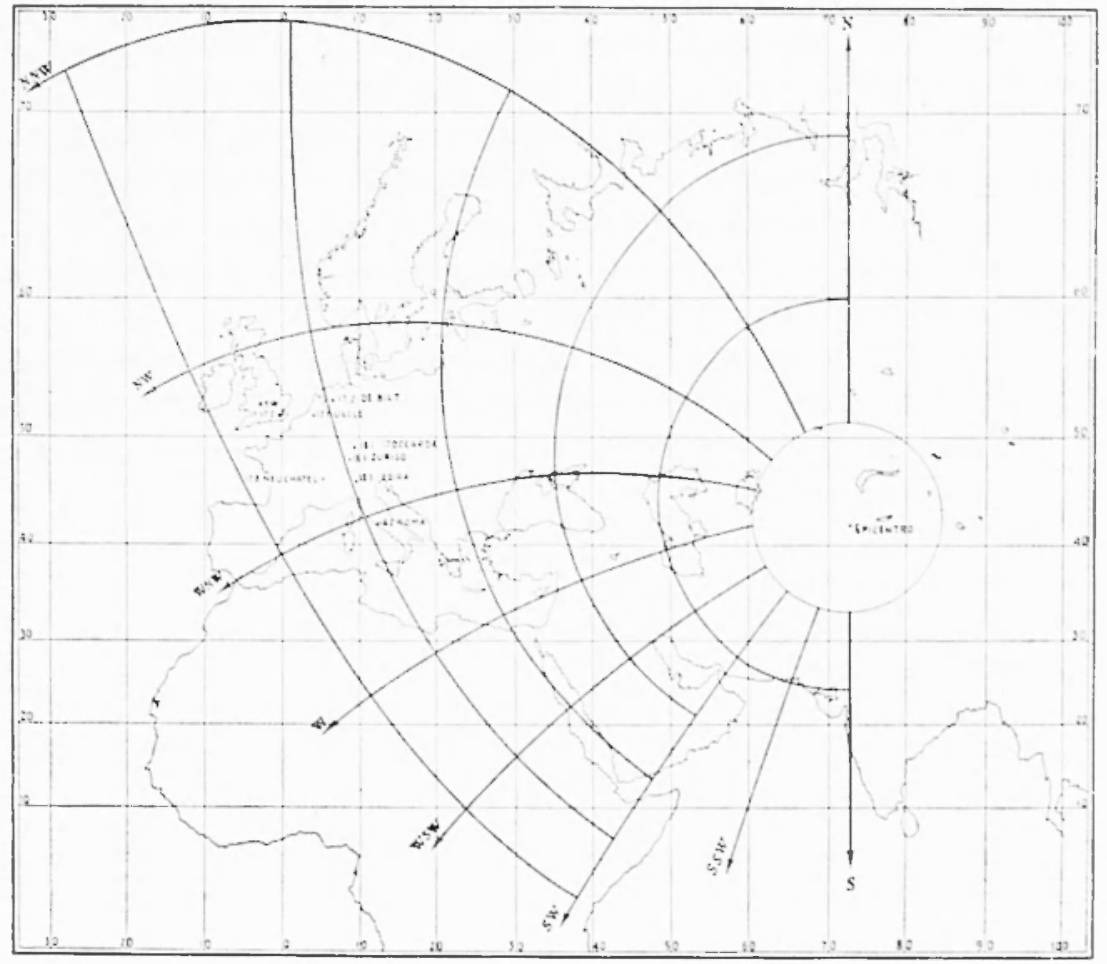

Fig. 2

volta oftenule le curve, lo spessote d dello strato si olliene direllamente dalla curva $C$ co se si la lipotesi che la velocita osservata per una certa lunghezza d'onta, sia la velociti di wruppo.

Nell'applicazione del metodo allo strato del pranito ho preferito usare i valori consigliati da Rohrbach $c_{0}=3,1, c^{\prime}=4,1, \quad \varphi_{0}=2,7$, $e^{\prime}=3,0$ che conducono an un rapporto tra i cocflicienti di rigiditi 


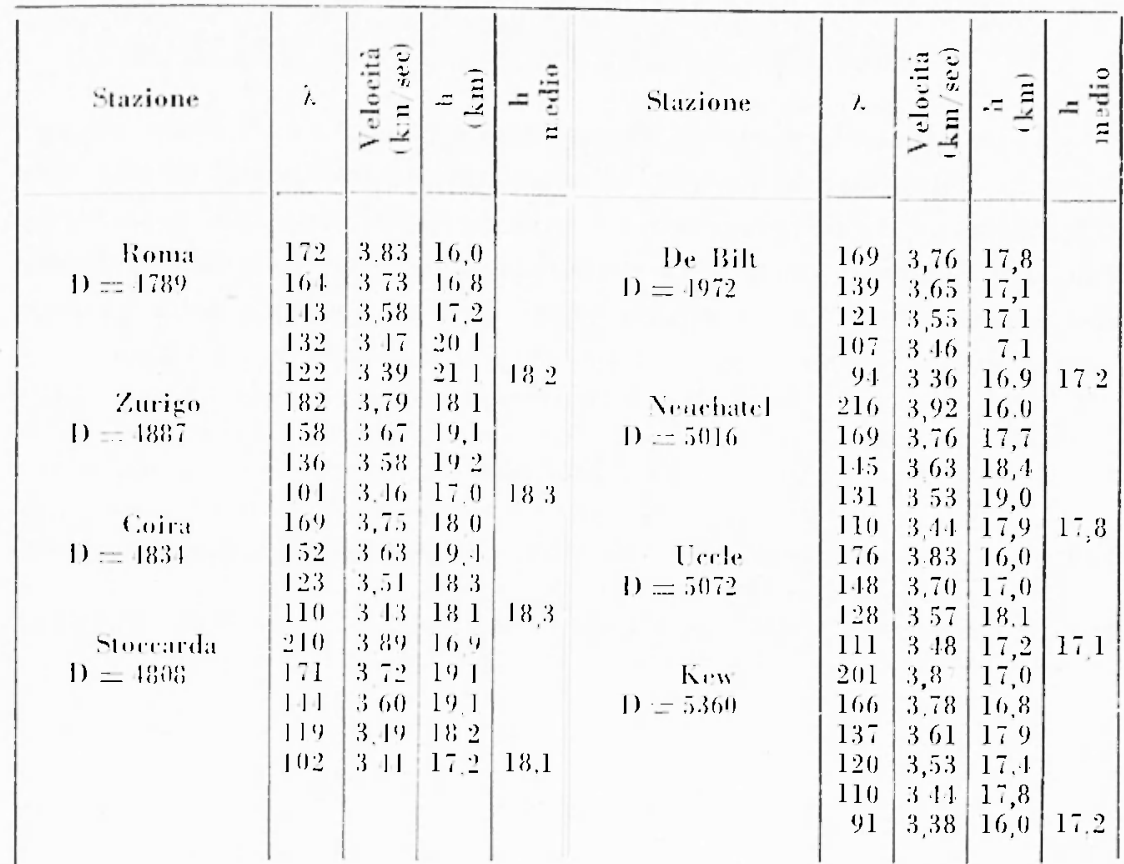

lelle due stratificazioni sovrappose pari a $\frac{u^{\prime}}{11}=1,9136$. Ho quindi calcolate le due cume fig. Il e delerminalo lo spessore dello strato

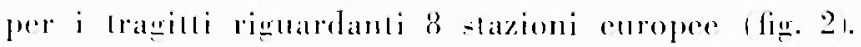

I risulati ollemuli rhe comblucono ad un valor medio di circa $18 \mathrm{~km}$-one riasumli nella labella $=11$ riporlala.

Faccio osservare the la determinazione is stat esemita nell’ipolesi che le sratificazione sollostante thatallot sia omogenea. In un successivo lavoro perio Jefleys ha consiglialo alcune modiliche da appor-

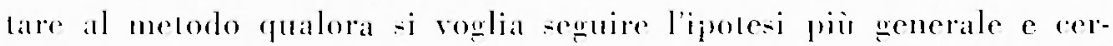
lamente piò vicina alla realla di una stratificazione sollostante non rispondente a questa caratleristica. Mi riprometlo di riesconire la determinazione tenende conto di fuesta oservazione.

Roma -.-. Istituto Nasionale di Geofisica - Gingno 1950. 


\section{RIASSITTO}

Deferminate le curre di dispersione per le onde di Loese seronder i valori consigliati da Rohrbach, si determina nellipotesi di una stratificazione inferiore omogeneea, lo speessore dello strato del granito per l'Eurasia centrale usando delle registrazioni del terremoto del Turtestan del 2 novembre 19.16. Si ottiene come medie un valore dello spessore pari a $18 \mathrm{hm}$ circe.

\section{BHBIOGRAHA}

Ronnbacin Watren: Uber die Dispersion seismicher Oberflächemechlen. Zeitselt. fur Geoplyss suppl. 1. 6 (1925).

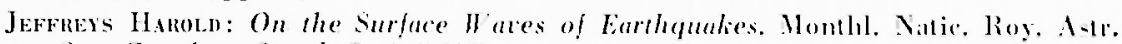
soce Geophys. Suppl. 1, 6 (19925). 